\title{
Prácticas de autocuidado en infecciones de transmisión sexual en adolescentes de población rural versus urbana
}

Ortiz-Hernández Karen Imelda*, Guerrero-Esparza Marisol Elizeth*, Montoya-Murillo Samantha Patricia*, Guerrero-Castillo Laura Helena*, Martínez-Contreras Carolina Elizabeth*, RodríguezHerrera Valeria Estibaliz*, Macías-Galaviz Ma Teresa**

\begin{tabular}{|c|c|}
\hline 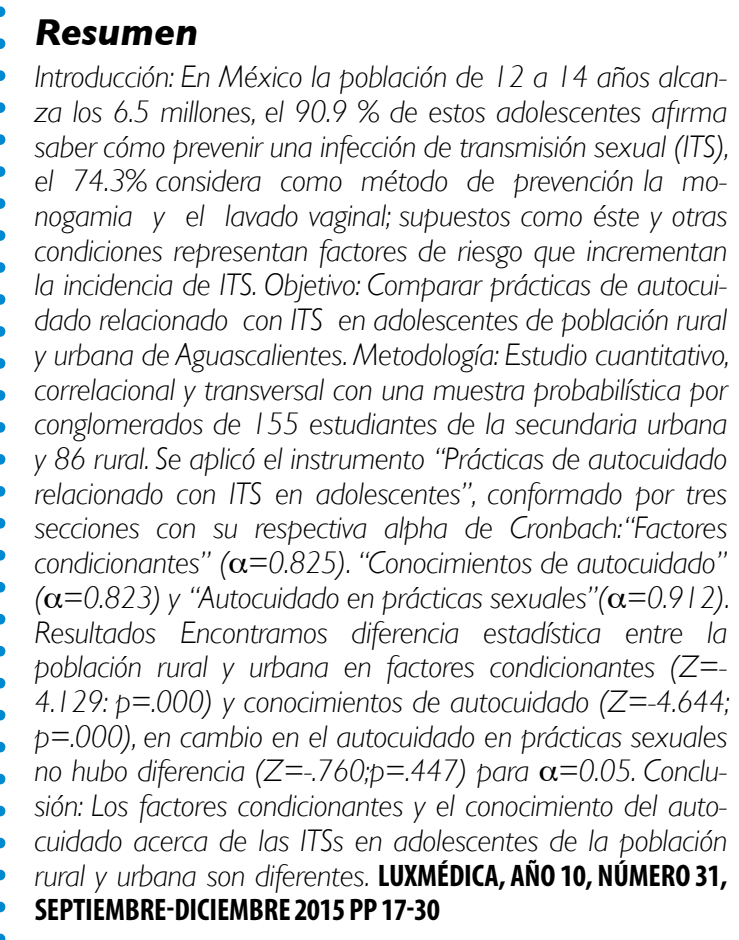 & 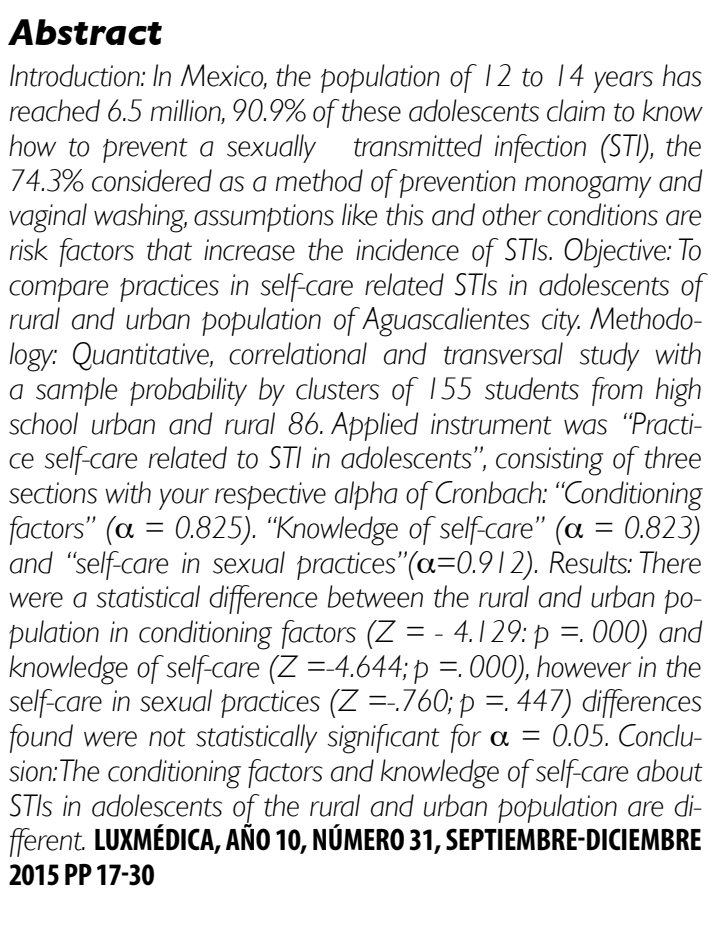 \\
\hline $\begin{array}{r}\text { Palabras Clave: Infecciones de Transmisiór } \\
\text { lescente }\end{array}$ & care \\
\hline
\end{tabular}

* Estudiantes de la Licenciatura en Enfermería de la Universidad Autónoma de Aguascalientes.

** MSPS profesora del Departamento de Enfermería del Centro de Ciencias de la Salud de la Universidad Autónoma de Aguascalientes.

Fecha de recibido: 10 de agosto 2015

Fecha de aceptación: 15 de septiembre 2015

Corresondencia: MSP Ma. Teresa Macías-Galaviz, Departamento de Enfermería, edificio 105, Centro de Ciencias de la Salud. Ciudad Universitaria. Universidad Autónoma de Aguascalientes, Avenida Universidad 940. Código postal 20131. Teléfono 9108437. Aguascalientes, Aguascalientes, México. Correo electrónico imeldahdz.2@hotmail.com 


\section{Introducción}

La juventud es una etapa clave en la vida de las personas puesto que en ella se toman decisiones que delimitan el actuar en la vida adulta, entendiendo a la adolescencia como un periodo transitorio donde se inicia la madurez fisiológica (con el desarrollo de las funciones sexuales y reproductivas), se forja la personalidad, afina su identidad, conforma su sistema de valores y con ello adoptar la capacidad de autocuidado en todas las esferas, etapa que culmina con la madurez social, esta última dada por indicadores subjetivos como la emancipación familiar, conclusión educativa e inicio de la vida laboral ${ }^{1}$. Orem contempla que el autocuidado es la práctica de actividades que los individuos inician y realizan en su propio beneficio para el mantenimiento de la vida, la salud y el bienestar. Se define el concepto auto como la totalidad de un individuo (incluyendo necesidades físicas, psicológicas y espirituales), y el concepto cuidado como la totalidad de actividades que un individuo inicia para mantener la vida y desarrollarse de una forma que sea normal para él. El cuidado se inicia voluntaria e intencionadamente por los individuos principalmente en la etapa de adolescencia. El autocuidado es un fenómeno activo que requiere que las personas sean capaces de usar la razón para comprender su estado de salud, y sus habilidades en la toma de decisiones para elegir un curso de acción apropiado. Es así como las personas desarrollan prácticas de autocuidado que se transforman en hábitos, que contribuyen a la salud y el bienestar. En México existe una problemática en cuanto autocuidado en infecciones de trasmisión sexual en la población adolescente. La población de 12 a 14 años alcanza un total de 6.5 millones de personas; distribuyéndose de la siguiente manera: 3.3 millones $(50.6 \%)$ corresponden al sexo masculino y 3.2 millones (49.4\%) al femenino ${ }^{2}$. El $90.9 \%$ de estos adolescentes dicen saber cómo prevenir una infección de transmisión sexual (ITS), de ellos el $74.3 \%$ considera que el método más eficaz para prevenir ITS es la monogamia y el lavado vaginal; encontrándose ahí un problema que incrementa la incidencia de ITS ${ }^{3}$.

Aguascalientes se posiciona como el estado en $5^{\circ}$ lugar de mayor incidencia a nivel nacional, de acuerdo al documento México Salud 2006. Por ello se vuelve programa prioritario para el estado la salud sexual y reproductiva del adolescente. En la Encuesta Nacional de Salud 2012 se encuentra que el $79.1 \%$ de los jóvenes de entre 12 a 19 años son sexualmente activos y de ellos reportan que el $14.7 \%$ de los hombres y $34.4 \%$ de las mujeres no utilizaron ningún método anticonceptivo en la primera relación sexual. "Es evidente por ejemplo que hoy en día los jóvenes mexicanos de entre 15 y 24 años se enfrenten a situaciones muy diversas que dependen en buena medida de su origen rural o urbano, de su estatus socioeconómico, condición de género e identidad cultural y étnica". ${ }^{1,4,5}$ Se considera que la población rural es vulnerable para 


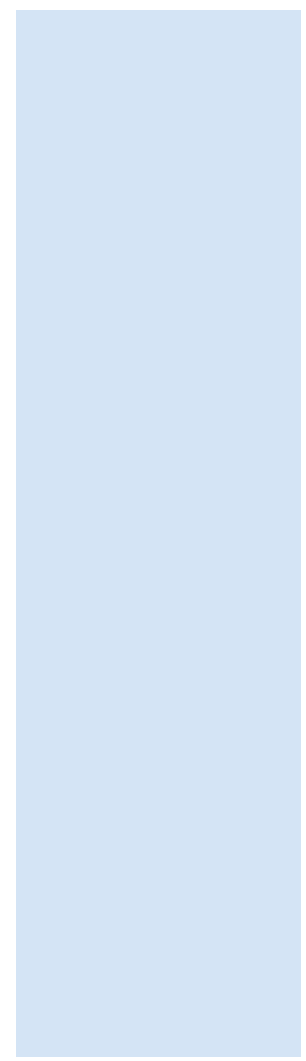

el desarrollo de ITS tomando en cuenta que en dichas comunidades los servicios de salud suelen ser limitados; en contra parte, dentro de las poblaciones urbanas hay más acceso a programas de salud y educación sexual. En un estudio previo, realizado en el municipio de Caldas, Colombia se plantea la problemática del alto índice de embarazos y como consecuencia de las ITS; se analizaron conocimientos y prácticas acerca de ITS y su relación a aspectos sociodemográficos en adolescentes, fue estudio descriptivo transversal, con una muestra no probabilística de 567 estudiantes entre 14 y 19 años de edad; la recolección de información se realizó a través de fuente primaria por encuesta anónima conformada por cuatro apartados: "Información sociodemográfica", "Conocimientos sobre vías de transmisión", "Métodos de protección, síntomas y riesgos", y "Actitudes de rechazo, estigma y protección contra ITS. Los resultados de relevancia son en protección, efectividad del condón, signos y síntomas de ITS reflejando conceptos errados; en conocimientos y prácticas se obtuvo nivel insatisfactorio y para el nivel de conocimientos se presentaron diferencias de acuerdo a la zona de procedencia, siendo mejor en estudiantes de la zona urbana. ${ }^{6}$

El objetivo de este trabajo fue comparar prácticas de autocuidado relacionado con ITS en adolescentes de población rural y urbana.

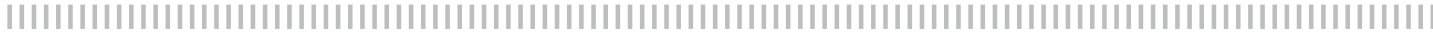

\section{Material y métodos}

El estudio fue de tipo descriptivo, transversal, correlacional. El universo de estudio lo conformó los 720 alumnos de la Escuela Secundaria General No. 20 y 102 de la Escuela Telesecundaria No. 173 del estado de Aguascalientes, la muestra fue de tipo probabilística por conglomerados con remplazo integrada por 155 participantes para la Secundaria General \# 20 "Siglo XXI" (Urbana); y de 86 participantes para la Telesecundaria "José Sixto V. "(Rural). Se incluyeron a los alumnos inscritos en el ciclo escolar 2014-2015, de 12 a 16 años y que se encontraban presentes el día de la realización de la encuesta en ambas instituciones. Se excluyeron a los alumnos que estaban fuera de edad de 12 a 16 años, a los que no quisieron participar y estudiantes con capacidades diferentes, fueron eliminados cinco instrumentos de la Escuela Secundaria Ge- neral No. 20 y tres instrumentos de la Escuela Telesecundaria José Sixto $\mathrm{V}$.

Se utilizó el instrumento exprofeso denominado "Prácticas de Autocuidado en Infecciones de Transmisión Sexual en Adolescentes" ("PAITSA") para comparar el Autocuidado Relacionado con ITS en adolescentes de población rural y urbana del estado de Aguascalientes. Fue piloteado en 34 estudiantes de secundaria validado con la alpha de Cronbach cuyo resultado fue una $\alpha=0.85$.

El instrumento consta de 29 ítems divididos en tres secciones: "Factores básicos condicionantes", "conocimientos de autocuidado" y "autocuidado en prácticas sexuales". En la segunda y tercera sección se toma la escala de Likert que se encuentra en el libro de resultados de enfermería (NOC) en los resultados correspondientes de conocimiento sobre la salud y autocuidado. 
Factores Básicos Condicionantes:

- Alto = 10-13 Puntos

- Medio=14-17 Puntos

- Bajo= 18-20 putos

\section{Conocimientos de Autocuidado}

- Ningún conocimiento $=26-23$ puntos

- Conocimiento escaso = 22-20 puntos

- Conocimiento moderado=19-17 puntos

- Conocimiento extenso $=16-13$ puntos

\section{Autocuidado en Prácticas Sexuales}

- Gravemente comprometido= 7-9 puntos

- Moderadamente comprometido=10-12 puntos

- Levemente comprometido=13-14 puntos

- No comprometido= 22-24 puntos

Previo a la aplicación del instrumento diseñado, se solicitó la participación voluntaria del entrevistado, haciéndole saber los fines de la información a recolectar y la completa privacidad de su persona. Cada participante dio su consentimiento informado.

El análisis estadístico se realizó con el SPSS (Statistical Package for the Social Sciences) versión 21. Las variables en estudio fueron sometidas a comparación entre ambas poblaciones (rural y urbana), examinadas en los apartados del instrumento aplicado (conocimientos de autocuidado, factores condicionantes, autocuidado en prácticas sexuales). Dicha comparación se elaboró a través de la prueba de U MannWhitney para un nivel de confianza del $95 \%$.

|I||||||||||||||||||||||||||||||||||||||||||||||||||||||||||||||||||||||||||||||||||||||||||||||||||||||||||||||||||||||||||||||

\section{Resultados}

Se encuestaron a 242 adolescentes, estudiantes de nivel secundaria, 86 provenientes de zona rural y 156 de zona urbana; de los cuales $48.7 \%$ fueron mujeres, y $51.30 \%$ hombres, con una media de edad de 13 años [intervalo de confianza (IC) del 95\%; límites de 11-16 años]. La distribución por edad (años cumplidos) fue de $27.98 \%$ para 12 años, 31.61\% 13 años, $30.05 \% 14$ años, 9.84\% 15 años y el $0.52 \%$ para 16 años (figura 1 ).

\section{Figura ||}

\section{Distribución por edad de los adolescentes}
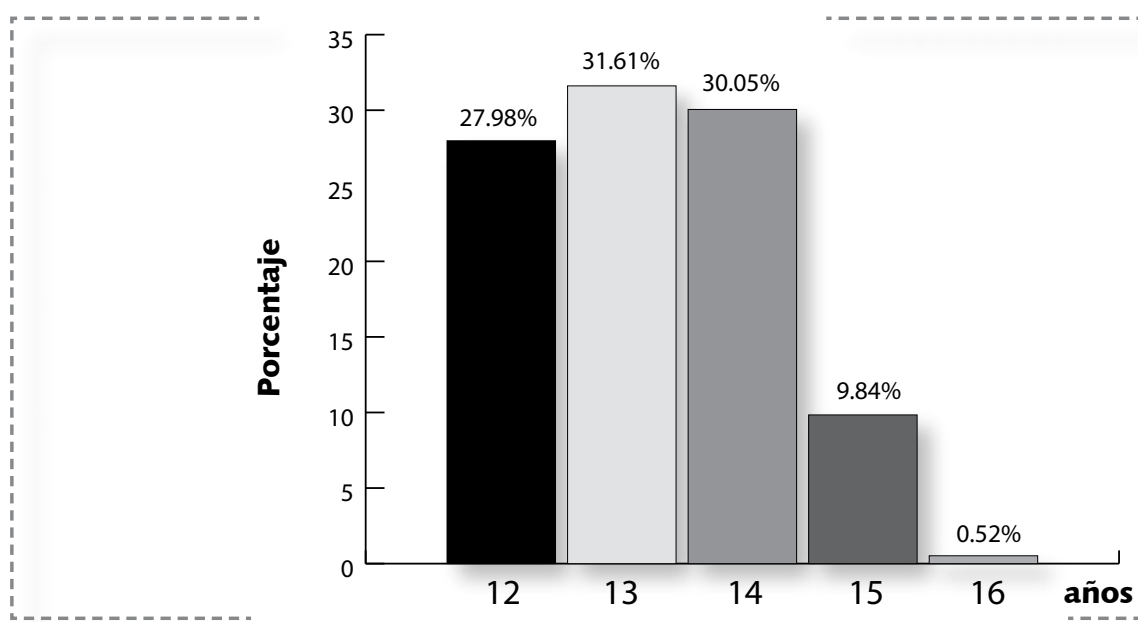

Fuente: Evaluación de las prácticas de autocuidado relacionado con ITS en adolescentes” PAITSA”. Febrero 2015. 
La distribución por grados sin distinción de sexo y zona poblacional es de $32.64 \%$ en el primer grado, $34.72 \%$ en segundo grado y $32.64 \%$ en el tercer grado (tabla 1 )

\section{Tabla I}

Distribución por año académico de nivel secundaria

\begin{tabular}{|lllll|}
\hline POBLACIÓN & SEXo & $\mathbf{1}^{\circ}$ & $\mathbf{2}^{\circ}$ & $\mathbf{3}^{\circ}$ \\
Rural & Femenino & $29 \%$ & $32 \%$ & $39 \%$ \\
\hline & Masculino & $35 \%$ & $38 \%$ & $27 \%$ \\
\hline Urbana & Femenino & $27 \%$ & $36 \%$ & $37 \%$ \\
\hline & Masculino & $39 \%$ & $33 \%$ & $28 \%$ \\
\hline
\end{tabular}

Fuente: Evaluación de las prácticas de autocuidado relacionado con ITS en adolescentes" PAITSA".

Febrero 2015.

El consumo de bebidas embriagantes, se encontró en mayor incidencia dentro de la zona rural ya que casi la mitad de los encuestados (47\%) contestó que sí ha consumido alcohol, mientras que sólo el $29 \%$ de los adolescentes de la zona urba- na hicieron la misma aseveración. Mismo fenómeno se observó en el consumo de drogas; un $27 \%$ de los adolescentes de la zona rural afirmaron haber consumido algún tipo de droga, frente a un $7 \%$ de la zona urbana (Figura 2).

\section{Figura 2}

\section{Distribución del consumo de drogas y alcohol}
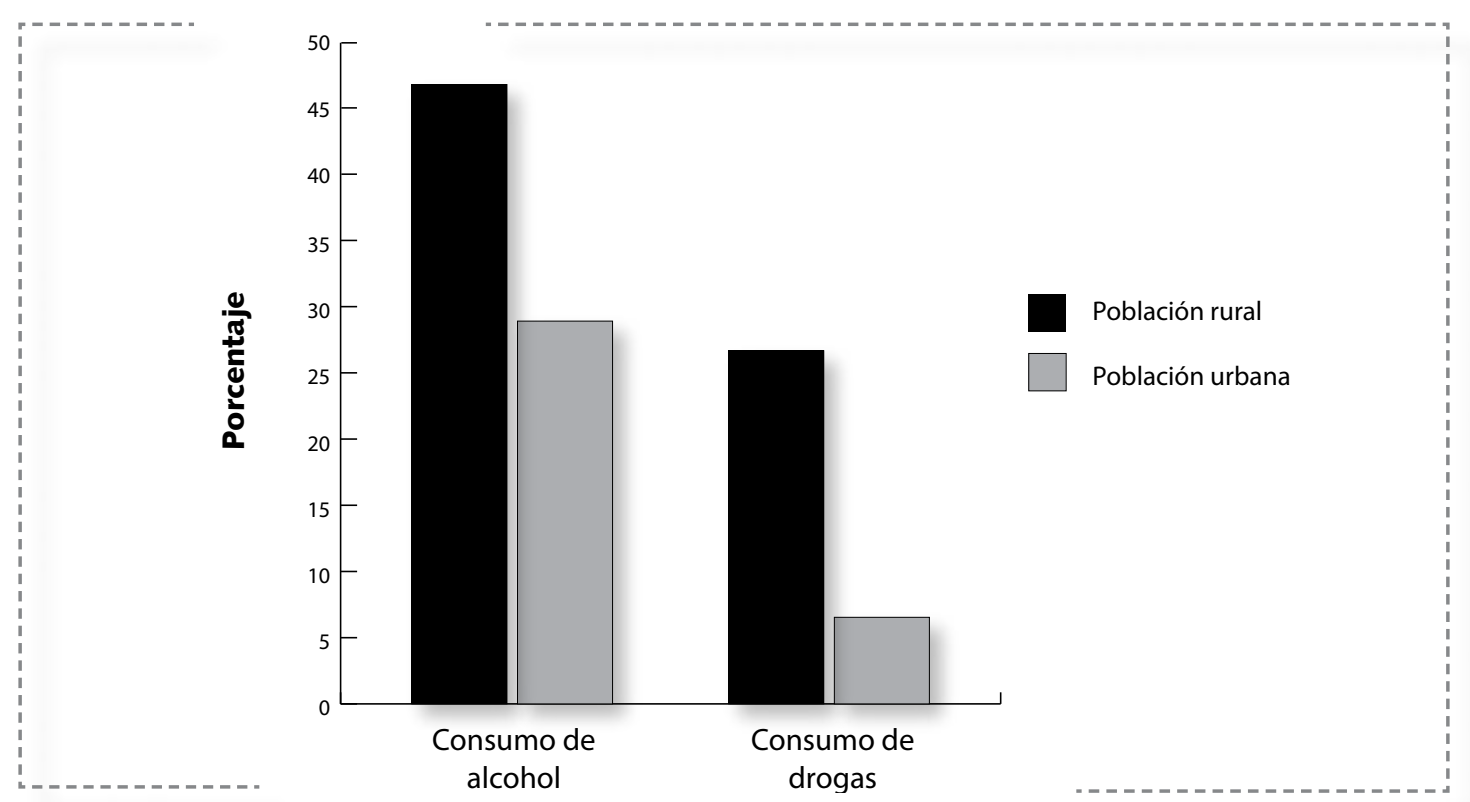

Fuente: Evaluación de las prácticas de autocuidado relacionado con ITS en adolescentes" PAITSA”. Febrero 2015. 
El $40 \%$ de los encuestados de la zona rural han recibido información sobre ITS por parte del personal de salud, en con-

\section{Tabla 3}

\section{Fuentes de información sobre ITS: Zona rural.}

\begin{tabular}{|ll|}
\hline Educación acerca de ITS de parte de... & $\%$ \\
Padres & 65 \\
\hline Amigos & 50 \\
\hline Personal de salud & 40 \\
\hline
\end{tabular}

traste con la zona urbana sólo el $29 \%$ hace referencia a dicha fuente (tablas 2 y 3 ).

\section{Tabla 4}

\section{Fuentes de información sobre ITS: Zona urbana.}

\begin{tabular}{|ll|}
\hline $\begin{array}{l}\text { Educación acerca de ITS de parte de... } \\
\text { Padres }\end{array}$ & \% \\
\hline Amigos & 65 \\
\hline Personal de salud & 27 \\
\hline
\end{tabular}

Fuente: Evaluación de las prácticas de autocuidado relacionado con ITS en adolescentes" PAITSA”. Febrero 2015.

En la zona rural el 15\% identificó a las ITS como infecciones ocasionadas por contacto homosexual exclusivo o por picadura de mosquito, en contraste, en la zona urbana el $95 \%$ identificó el término ITS como aquella infección causada por contacto sexual, a su vez en la zona rural el $20 \%$ asumió como métodos de prevención los lavados vaginales y anticonceptivos orales (Figura 3).

\section{Figura 3}

Distribución de la opinión que tienen los encuestados acerca del mecanismo de transmisión de las ITS

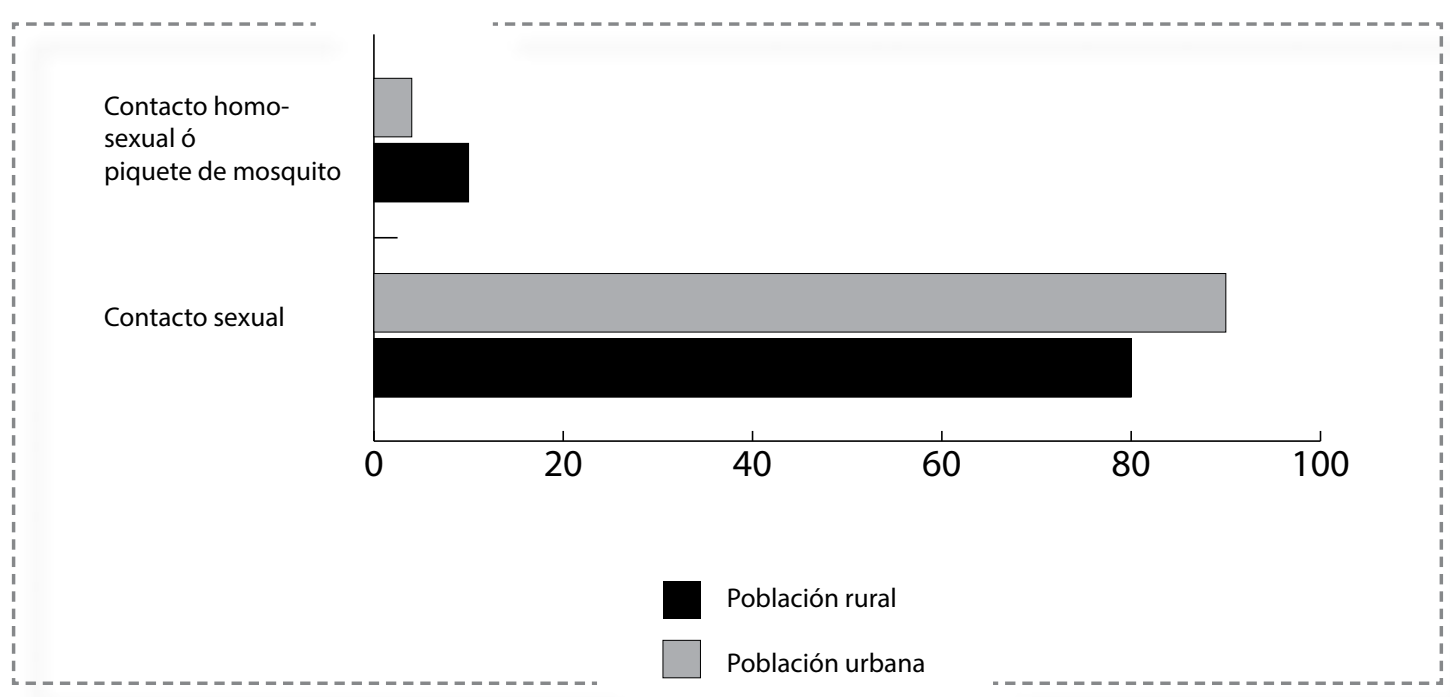

Fuente: Evaluación de las prácticas de autocuidado relacionado con ITS en adolescentes" PAITSA". Febrero 2015. 
Los adolescentes estudiados no identifican que toda práctica sexual sin protección representa riesgo de contagio de ITS (sexo anal, vaginal y oral sin protección). Alre- dedor de la mitad poblacional de la zona rural y una cuarta parte de la zona urbana, perciben el riesgo al tener contacto vía vaginal (figura 4).

\section{Figura 4}

Distribución de la opinión de los adolescentes acerca de tipo de contacto sexual y riesgo de ITS

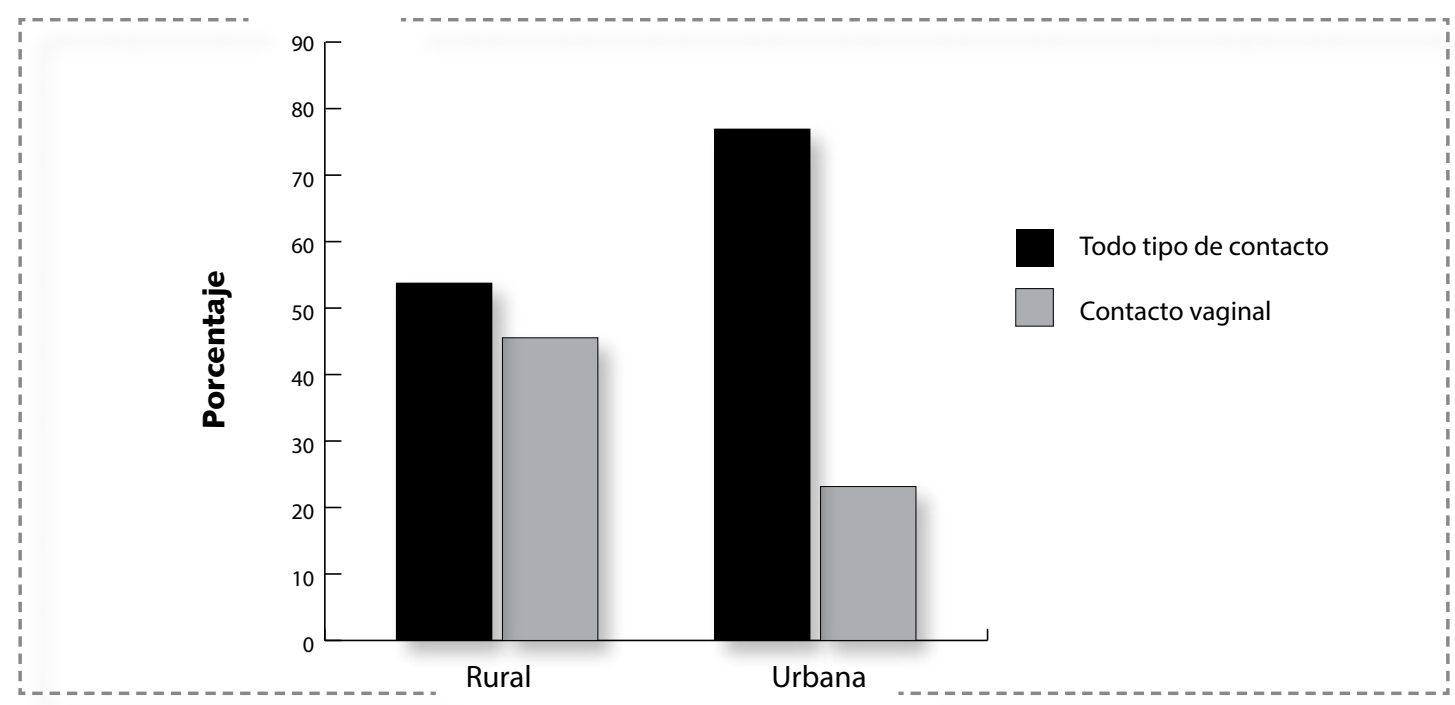

Fuente: Evaluación de las prácticas de autocuidado relacionado con ITS en adolescentes" PAITSA". Febrero 2015.

De los 242 adolescentes encuestados solo $8.29 \%$ asegura tener vida sexual activa con una distribución tres veces mayor en la población rural en relación a la urbana (tabla 4).

\section{Tabla 4}

\section{Proporción de los adolescentes sexualmente activos}

\begin{tabular}{|ll|}
\hline POBLACIÓN & SEXUALMENTE ACTIVOS \\
\hline Población general & $8.29 \%$ \\
\hline Rural & $12 \%$ \\
\hline
\end{tabular}

Fuente: Evaluación de las prácticas de autocuidado relacionado con ITS en adolescentes" PAITSA”. Febrero 2015.

La edad mínima de inicio vida sexual activa en ambas poblaciones es de 13 años, en la zona rural la mitad de los adolescentes comienzan su vida sexual a esa edad, mientras que en la población urbana lo hace un año después, es decir a los 14 años (figura 5). 


\section{Figura 5}

\section{Distribución de la edad de inicio de la vida sexual en los adolescentes estudiados}

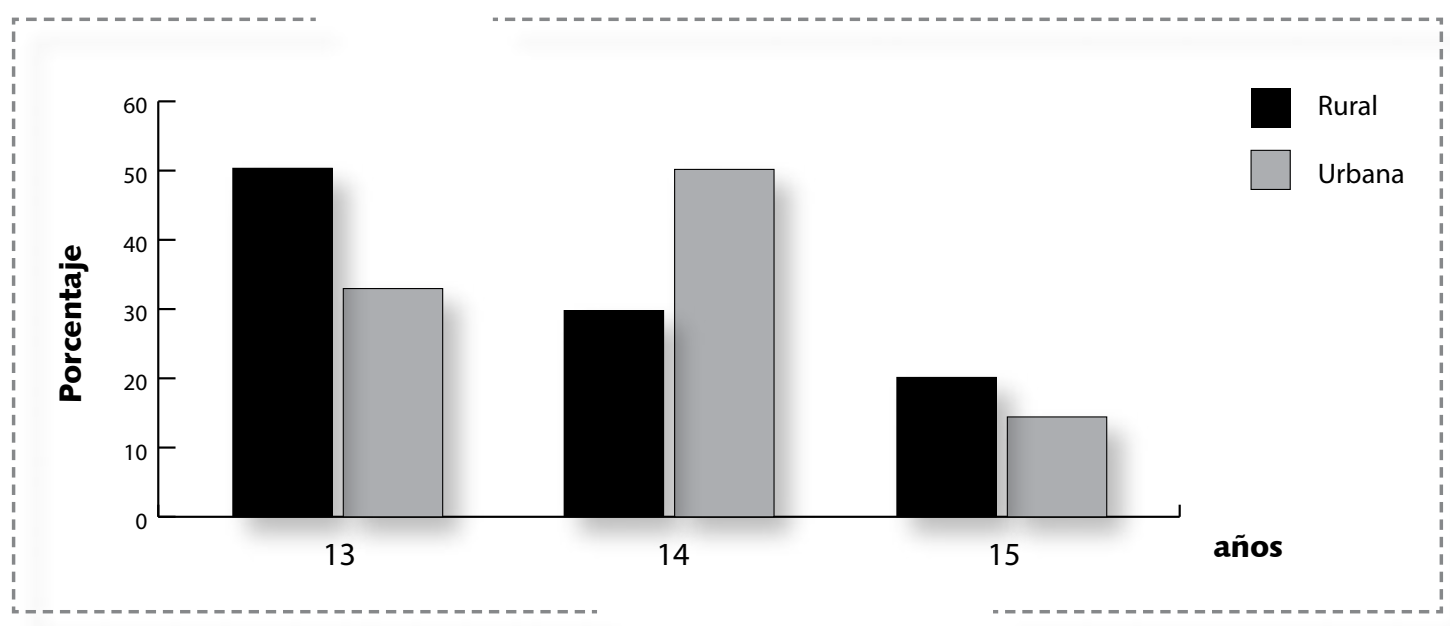

Fuente: Evaluación de las prácticas de autocuidado relacionado con ITS en adolescentes” PAITSA”. Febrero 2015.

El uso del condón predomina en el inicio tiempo la frecuencia disminuye en ambas de vida sexual activa y conforme pasa el poblaciones (figura 6).

\section{Figura 6}

\section{Uso del condón en población rural y urbana.}

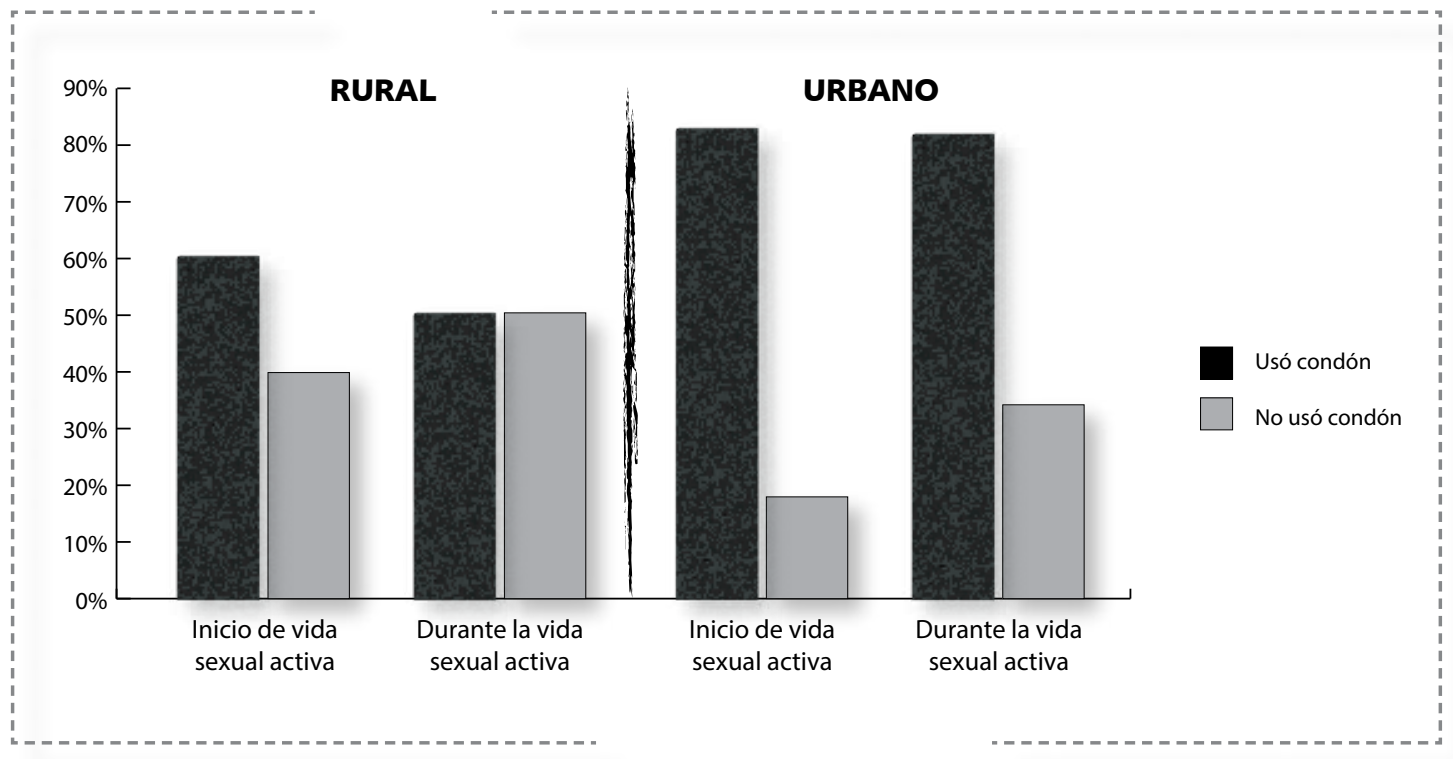

Fuente: Evaluación de las prácticas de autocuidado relacionado con ITS en adolescentes" PAITSA”. Febrero 2015. 
Para la sección de "Factores Condicionantes", se obtuvo en la prueba MannWhitney un valor de $Z=-4.129$ y $p=$ 0.000 para $\alpha=0.05$, para el apartado de factores condicionantes. Por lo tanto estadísticamente se establece que los adolescentes de la población rural tienen diferente nivel de factores condicionantes de autocuidado relacionado con ITS que los adolescentes de la población urbana. En "Conocimientos de Autocuidado", se obtuvo en la prueba Mann-Whitney un valor de $Z=-4.644$ y $p=0.000$ para $\alpha=0.05$. Por lo tanto estadísticamente se establece que los adolescentes de la población rural tienen diferente nivel de conocimiento de autocuidado relacionado con ITS que los adolescentes de la población urbana. En el Autocuidado en prácticas sexuales, se obtuvo en la prueba Mann-Whitney un valor de $Z=-0.760$ y $p=0.447$, para $\alpha=0.05$ Por lo tanto estadísticamente se establece que los adolescentes de la población rural tienen igual nivel de autocuidado en prácticas sexuales relacionado con ITS que los adolescentes de la población urbana.

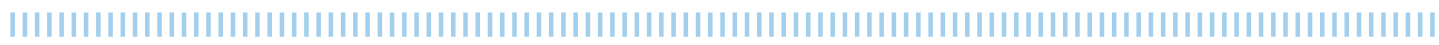

\section{Discusión}

La media de edad de los adolescentes observados es de trece años, misma cifra que marca el promedio de inicio de vida sexual activa en la zona rural, con una variación de un año para los adolescentes de la zona urbana donde el promedio es de 14 años, en comparativo a la década anterior encontramos que la edad media de inicio de la vida sexual se ha centralizado puesto que la edad promedio en la zona rural oscilaba en los 11 años contra 15 años para el caso de la urbana. ${ }^{4}$

En relación a las fuentes de las que el adolescente obtiene información acerca de las ITS, cabe destacar que en ambas poblaciones el $65 \%$ ha recibido información por parte de sus padres, lo que resalta es que menos de la tercera parte de los encuestados afirman haber recibido información por parte del personal de salud, con una diferencia de 10 puntos entre las zonas urbana y rural, siendo mayor el acercamiento del personal de salud en la última, esto se puede asociar de manera directa con el hecho que en dichas zonas el personal de salud tiene un acercamiento más estrecho con la población, dado por el reducido número de habitantes y los programas que se llevan a cabo en los centros de salud; en Aguascalientes existen 83 Centros de Salud coordinados en tres jurisdicciones, 61 de ellos distribuidos en las zonas rurales del estado dentro de los que se llevan a cabo programas como salud sexual y reproductiva en adolescentes que exigen por sus características el abordaje directo de las familias de la zona. A pesar del acercamiento de la población rural al sistema de salud, las prácticas sexuales y conocimientos básicos en la materia de los adolescentes de la zona, resultan más riesgosos a los llevados a cabo en la zona urbana, asumiendo que la dinámica social y familiar tiene importante influencia en las prácticas y la baja percepción de riesgo independientemente de que en ambas poblaciones se cuente con el mismo nivel académico.

De los 242 adolescentes encuestados el $90 \%$ tiene un concepto acertado sobre que son las ITS, sin embargo el $39 \%$ de los adolescentes de la zona urbana y, el $41 \%$ de la zona rural no logran identificar cuáles son las principales infecciones, a su vez en esta última, casi la mitad (46\%) no reconoce que toda práctica sexual sin protección representa un riesgo de contagio; al hablar de medidas de protección el conocimiento en relación a métodos 
es elevado ya que solo una décima parte considera como métodos de protección ante ITS los lavados vaginales y el uso de pastillas anticonceptivas, resultado 7 veces menor al arrojado por la Encuesta Nacional de Salud y Nutrición 2012 (ENSANUT 2012), lo que indica que los conocimientos errados y mitos se han ido reduciendo entre los jóvenes mexicanos con un nivel de instrucción adecuado a su edad cronológica.5 Aún más preocupante es que casi la quinta parte de la población en estudio (en igual proporción en ambas zona), identifica a la infección por el virus de la inmunodeficiencia humana (VIH) como una ITS curable, en la última década la mitad de los nuevos casos de $\mathrm{VIH} /$ SIDA a nivel mundial se registró en el grupo de edad de 10 a 24 años (ONUSIDA 2010), evidentemente demuestra la vulnerabilidad de este grupo ante el contagio del virus, pues al considerarlo como un padecimiento curable se crea una visión alterada del riesgo.

Solo el 8\% de la población general afirmó tener una vida sexual activa donde la media de inicio es de 13 años, con un año de retardo (14 años) para el caso de la población urbana, así mismo la población rural triplica en proporción a la población urbana, por lo que se puede inferir a la primera bajo situación de mayor riesgo, por dichas prácticas.

Dato curioso es el resultado en el uso de condón la Organización Mundial de la Salud (OMS) declara al preservativo como método anticonceptivo y de protección de primera elección, la décima parte de la población es sexualmente activa de la que más de la mitad afirma su uso en el primer encuentro sexual, esta tendencia se ve disminuida en el transcurso de los encuentros, hecho íntimamente relacionado con la baja percepción del riesgo ya que el adolescente adquiere cierto grado de confianza conforme continua su práctica sexual, asumiendo conductas de "invulnerabilidad". Del total de adolescentes sexualmente activos en las poblaciones en estudio 70\% reportó haber utilizado preservativo en la primera relación sexual, solo 6 puntos menos en comparativo con los datos obtenidos a nivel nacional en el 2012. ${ }^{5}$

Observamos diferencias estadísticamente significativas al comparar los factores básicos condicionantes entre la población rural y urbana, $(p<0.05)$. Utilizando la $U$ de Mann Whitney encontramos que existen diferencias estadísticamente significativas en el nivel de conocimientos de autocuidado sobre ITS entre la población rural y urbana, ( $p<0.05$ ), resultados que coinciden con un estudio realizado en Caldas, Colombia en el cual se analizaron conocimientos, prácticas acerca de ITS y su relación a aspectos sociodemográficos en adolescentes; los resultados de relevancia son en protección, efectividad del condón, signos y síntomas de ITS reflejando conocimientos errados, en conocimientos y prácticas se obtuvo nivel insatisfactorio y para el nivel de conocimientos se presentaron diferencias de acuerdo a la zona de procedencia, siendo mejor en estudiantes de la zona urbana. ${ }^{6}$

Por último, no encontramos diferencias estadísticamente significativas entre el nivel de autocuidado en función a las prácticas sexuales de las poblaciones rural y urbana, situación similar a la reportada por otros autores, donde la población sexualmente activa de zona rural lleva a cabo menos prácticas de autocuidado que la urbana ${ }^{7,8}$ 


\section{Conclusiones}

Se entiende al adolescente como un grupo vulnerable, por lo que se considera como una población en riesgo independientemente de la zona (rural o urbana) en la que se encuentre, dicha vulnerabilidad dada en primera instancia por la baja percepción del riesgo, los conocimientos errados en materia de salud sexual y reproductiva, así como las propias practicas sin protección. Esto asociado a los fenómenos que se observan en las prácticas de autocuidado que llevan los adolescentes independientemente de la zona de residencia y los conocimientos que se tengan del tema. La importancia de programas de salud eficaces a edades más tempranas modifica estilos de vida y propician conductas saludables, las intervenciones deben abocarse de manera introductoria reforzando los conocimientos, sobre todo en la zona rural, pero de manera precisa desarrollando la conciencia sexual del adolescente para que optimice los conceptos que le fueron brindados y de esta manera fortalecer el autocuidado de manera individual, repercutiendo de manera comunitaria.

\section{Bibliografía}

1. Cruz C, Ramos U, Guía de prevención, diagnóstico y tratamiento de las ITS Dirigida a personal de servicios de salud. Primera edición, México, Fundación Mexicana para la Salud, 2011.

2. Infecciones de Transmisión Sexual (ITS), VIH/SIDA en Mujeres en el Estado de Aguascalientes: Estudio desde la Perspectiva de Género [En línea] .Aguascalientes, México:IAM;2011[Consultado el 25 de marzo de 2014] Disponible en:

http://cedoc.inmujeres.gob.mx/ftpg/Aguascalientes/agsmeta11.pdf

3. CENSIDA. Centro Nacional para la Prevención y el Control del VIH y el sida [En línea].CENSIDA, México: Secretería de Salud; 2014[Consultado 25 de marzo 2014].Disponible en: http://www.censida. salud.gob.mx/interior/cifras.html

4. Enfermedades de transmisión sexual y adolescencia: Generalidades y prevención. Manual de la Secretaría de Salud. [Consultada 25 de marzo de 2014]. Disponible en: http://www.sec.es/area_cientifica/ manuales_es/salud_reproductiva/15\%20Salud\% 20 reproducti va\% 20 e.pdf
5. Organización Mundial de la Salud [En línea], Infecciones de Transmisión Sexual, Nota Descriptiva No110, Centro de prensa.c20014; 2014, [Consultado 25 de Marzo de 2014]. Disponible en: http:// www.who.int/mediacentre/factsheets/fs110/es/

6. Cardona A, Arboleda C, Rosero C. Conocimientos, actitudes y práctiucas acerca de infecciones de transmisión sexual en adolescentes en el municipio de Caldas, Colombia. Salud Uninorte (en línea), 2013 (consultado 18 de dic 2014), disponible en: http:// www.redalyc.org/pdf/817/81730430009.pdf

7. Gayet Cecilia, Júarez Fátima, Pedroza Laura, Magis Carlos. Uso del condón entre adolescentes mexicanos para la prevención de las infecciones de trnamisión sexual. Salud pública de Méx (revista en internet) 2003(citado enero 2015) disponible en http:// www.scielo.org.mx

8. Encuesta Nacional de la Dinámica Demográfica, Principales Indicadores de Salud reproductiva, Reporte [versión en línea], México [última actualización 2013],[consultada en diciembre del 2014],2010 disponible en: http://www.portal.conapo.gob.mx/ index. php?option $=$ com_content $\&$ view $=$ article $\& i d=$ 455\&Itemi $d=15$ 


\section{"Instrumento de evaluación (PAITSA)"}

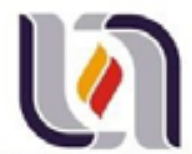

POBLACIÓN

UחIUERSIDaD auTonoma

DE aGuascalientes

"EVALUACIÓN DE LAS PRÁCTICAS DE AUTOCUIDADO RELACIONADO CON ITS EN ADOLESCENTES" "PAITSA"

\section{DATOS GENERALES}

\begin{tabular}{|l|l|l|l|l|l|l|}
\hline \multicolumn{2}{|l|}{ ESCUELA: } & \multicolumn{5}{l|}{} \\
\hline EDAD: & & SEXO: & F & M & TURNO: & \\
& & & & & & \\
\hline
\end{tabular}

GRADO:

\begin{tabular}{|l|l|l|l|}
\hline $1^{\circ}$ & $2^{\circ}$ & $3^{\circ}$ & LUGAR DONDE VIVE: \\
\hline
\end{tabular}

FACTORES BASICOS CONDICIONANTES:

Conteste la siguiente información marcando solamente una respuesta:

\begin{tabular}{|c|c|c|c|}
\hline & & VERDADERO & FALSO \\
\hline 1 & Ha consumido alcohol & & \\
\hline 2 & $\begin{array}{l}\text { Ha consumido algún tipo de } \\
\text { droga (Tabaco, marihuana, } \\
\text { etc.) }\end{array}$ & & \\
\hline 3 & $\begin{array}{c}\text { Vive con otra persona que no } \\
\text { sean sus padres }\end{array}$ & & \\
\hline 4 & Además de estudiar trabaja & & \\
\hline 5 & Se siente inseguro en casa & & \\
\hline 6 & $\begin{array}{c}\text { Considera que la relación con } \\
\text { sus padres es buena. }\end{array}$ & & \\
\hline 7 & $\begin{array}{l}\text { Ha recibido información } \\
\text { sobre ITS por parte de sus } \\
\text { padres }\end{array}$ & & \\
\hline 8 & $\begin{array}{c}\text { Ha recibido información } \\
\text { sobre ITS por parte de sus } \\
\text { amigos. }\end{array}$ & & \\
\hline 9 & $\begin{array}{l}\text { Ha recibido información } \\
\text { sobre ITS por parte del } \\
\text { personal de salud. }\end{array}$ & & \\
\hline 10 & $\begin{array}{l}\text { Las ITS solo se contagian a } \\
\text { mayores de } 20 \text { años. }\end{array}$ & & \\
\hline
\end{tabular}

CONOCIMIENTOS SOBRE AUTOCUIDADO: Conteste las siguientes preguntas subrayando la respuesta que crea que es la correcta, solo una de ellas es la correcta: 


\begin{tabular}{|l|l|}
\hline 11 & $\begin{array}{l}\text { ¿Qué son las ITS? } \\
\text { a) Infección que se da por contacto homosexual. } \\
\text { b) Infección que se da por contacto sexual. } \\
\text { c) Infección que se da por picadura de mosquito. }\end{array}$ \\
\hline 12 & $\begin{array}{l}\text { ¿Cuáles son los métodos de protección contra las ITS? } \\
\text { a) Preservativo (condón) } \\
\text { b) Patillas anticonceptivas. } \\
\text { c) Lavado de la zona genital al terminar la relación sexual }\end{array}$ \\
\hline 13 & $\begin{array}{l}\text { ¿Cuáles de éstas son infecciones de trasmisión sexual? } \\
\text { a) Neumonía, varicela. } \\
\text { b) Hipertiroidismo, asma. }\end{array}$ \\
\hline
\end{tabular}

\begin{tabular}{|c|c|}
\hline & c) Sífilis, papiloma, gonorrea, herpes. \\
\hline 14 & $\begin{array}{l}\text { ¿Cuál de las siguientes es una consecuencia a largo plazo de las ITS? } \\
\text { a) Dolor de cabeza } \\
\text { b) Sueño } \\
\text { c) Vómito } \\
\text { d) Infertilidad }\end{array}$ \\
\hline 15 & $\begin{array}{l}\text { ¿Cuáles son las formas de contagio de las ITS? } \\
\text { a) Fluido vaginal, sangre y semen } \\
\text { b) Sudor, lágrimas y semen. } \\
\text { c) Sangre, sudor y saliva. }\end{array}$ \\
\hline 16 & $\begin{array}{l}\text { ¿Qué tipos de condón conoces? } \\
\text { a) De sabores, texturizados y masculinos. } \\
\text { b) Masculino y femenino. } \\
\text { c) Masculino, femenino, de sabor, texturizado de color. } \\
\text { d) Ninguno }\end{array}$ \\
\hline 17 & $\begin{array}{l}\text { ¿Dónde no debe guardarse el condón? } \\
\text { a) En la cartera } \\
\text { b) En la bolsa } \\
\text { c) En la mochila } \\
\text { d) Ninguna de las anteriores. }\end{array}$ \\
\hline 18 & $\begin{array}{l}\text { ¿El condón puede ser usado más de una vez? } \\
\text { a)Si } \\
\text { b)No }\end{array}$ \\
\hline 19 & $\begin{array}{l}\text { ¿Conoce el condón femenino? } \\
\text { a)Si } \\
\text { b)No }\end{array}$ \\
\hline 20 & $\begin{array}{l}\text { ¿Cuál de la siguientes ITS no es curable? } \\
\text { a) Sífilis } \\
\text { b) Gonorrea } \\
\text { c) VIH }\end{array}$ \\
\hline 21 & $\begin{array}{l}\text { ¿Si una mujer esta contagiada por ITS que síntomas presenta? } \\
\text { a) Vómito, diarrea, cólicos. } \\
\text { b) Flujo vaginal con olor feo, comezón en zona genital, fiebre. } \\
\text { c) Flujo vaginal con olor feo, dolor de cabeza, escalofrió. }\end{array}$ \\
\hline 22 & $\begin{array}{l}\text { ¿Si un hombre esta contagiado por ITS que síntomas presenta? } \\
\text { a) Vómito, diarrea, cólicos. } \\
\text { b) Secreción amarilla o verdosa en pene, comezón en zona genital, fiebre. } \\
\text { c) Secreción amarilla o verdosa en pene, dolor de cabeza, escalofrió. }\end{array}$ \\
\hline 23 & $\begin{array}{l}\text { ¿Cuáles de las siguientes prácticas sexuales sin protección representan un riesgo } \\
\text { para contraer ITS? } \\
\text { a) Vaginal } \\
\text { b) Oral } \\
\text { c) Anal } \\
\text { d) Todas } \\
\text { e) No se }\end{array}$ \\
\hline
\end{tabular}

\section{AUTOCUIDADO EN PRÁCTICAS SEXUALES}

Conteste las siguientes preguntas subrayando la respuesta correspondiente: 


\begin{tabular}{|l|l|l|}
\hline 24 & $\begin{array}{l}\text { ¿Usted ha tenido relaciones sexuales? } \\
\text { a)Si } \\
\text { b)No }\end{array}$ & \\
\hline Si su respuesta anterior fue si continúe con la encuesta & \\
\hline 25 & ¿A qué edad tuvo su primera relación sexual? & \\
\hline
\end{tabular}

\begin{tabular}{|l|l|l|}
\hline & $\begin{array}{l}\text { a)12 } \\
\text { b)13 } \\
\text { c)14 } \\
\text { d)15 }\end{array}$ & \\
\hline 26 & $\begin{array}{l}\text { ¿Con quién fue su primera relación sexual? } \\
\text { a) Amigo (a) } \\
\text { b) Conocido (a) } \\
\text { c) Novio (a) } \\
\text { d) Desconocido (a) }\end{array}$ & \\
\hline 27 & $\begin{array}{l}\text { ¿Uso condón en su primera relación sexual? } \\
\text { a)Si } \\
\text { b)No }\end{array}$ & $\begin{array}{l}\text { ¿Cuántas relaciones sexuales ha tenido? } \\
\text { b)Una } \\
\text { c)Dos } \\
\text { d)Más de dos }\end{array}$ \\
\hline 28 & $\begin{array}{l}\text { ¿Usa preservativo (condón) al tener relaciones sexuales? } \\
\text { a)Si } \\
\text { b)No }\end{array}$ & \\
\hline 2
\end{tabular}

